\title{
Research Agenda for Antimicrobial Stewardship in the Veterans Health Administration
}

\author{
Katie J. Suda, PharmD, MS; ${ }^{1,2, a}$ Daniel J. Livorsi, MD, MSc; ${ }^{3,4, a}$ Michihiko Goto MD, MSc; ${ }^{3,4}$ Graeme N. Forrest, MD; ${ }^{5}$ \\ Makoto M. Jones, MD; $;^{6}$ Melinda M. Neuhauser, PharmD, MPH; $;^{7}$ Brian M. Hoff, PharmD; ${ }^{8}$ Dilek Ince, MD; $;^{4,8}$ \\ Margaret Carrel, PhD; ${ }^{9}$ Rajeshwari Nair, MBBS, PhD; ${ }^{3,4}$ Mary Jo Knobloch, PhD, MPH; ${ }^{10}$ Matthew B. Goetz, $\mathrm{MD}^{11}$
}

(See introductory commentary by Livorsi et al, pages 186-188.)

Antimicrobial use is a key contributor to increasing antimicrobial resistance. ${ }^{1}$ Antimicrobial stewardship has been shown to decrease inappropriate antimicrobial use and, in turn, may reduce antimicrobial resistance and Clostridium difficile infection (CDI). ${ }^{2}$ Antimicrobial stewardship can also improve clinical outcomes for patients. ${ }^{3}$

The Veterans Health Administration (VHA) is a leader in promoting antimicrobial stewardship. ${ }^{4-7}$ Since 2011, the VHA National Antimicrobial Stewardship Taskforce (ASTF) has provided guidelines and resources for stewardship implementation at individual VHA facilities. In 2014, the VHA mandated that every facility develop and maintain an antimicrobial stewardship program (ASP). The VHA ASP Directive tasked every VHA facility to have an ASP policy, to conduct an annual evaluation of ASP activities, and to have a provider and a pharmacist champion. ${ }^{6}$ There were no requirements for facilities to adopt any specific set of ASP activities, allowing individual facilities to decide which elements to implement based on their own focus areas and available resources. This mandate was among the first in the United States, preceding similar directives from the Joint Commission and the Centers for Medicare and Medicaid Services. ${ }^{8,9}$ Recently, VHA investigators have partnered with the Centers for Disease Control and Prevention (CDC) to make use of novel antimicrobial usage metrics, including the standardized antimicrobial administration ratio (SAAR). They have also utilized the robust VA Corporate Data Warehouse (CDW) to determine the effectiveness of ASPs, to evaluate outcomes, and to identify high-impact targets. ${ }^{6,7,10-12}$

As the largest integrated healthcare system in the United States, the VHA provides a unique opportunity to study strategies to improve antimicrobial prescribing and the effectiveness of these interventions. Herein, we describe the proceedings from a multidisciplinary conference focused on developing an agenda for antimicrobial stewardship research in inpatient and outpatient healthcare settings within the VHA (long-term care is described separately, see commentary by Evans CT, pages 210-213). The methods are described elsewhere (see preceding editorial). The research targets were designed specifically for health services researchers within the VHA, but our findings also have implications more broadly in private sector healthcare settings (Table 1).

\section{RECOMMENDATIONS}

\section{Inpatient Antimicrobial Stewardship}

Optimal structure of ASP teams. Antimicrobial stewardship programs led by infectious disease (ID) specialists (physicians and/ or pharmacists) have been successful at reducing unnecessary antimicrobial use and in improving appropriate use., ${ }^{2,6,13}$ However, due to staffing limitations, the implementation of stewardship across the continuum of health care is not feasible if an ID specialist must be the leader of every program. Many hospitals and nonacute residential care settings (eg, long-term care) lack and/or cannot afford ID specialists to assist with stewardship. Even at hospitals that have ID specialists involved in stewardship, limitations in both resources and time prevent routine intervention on every antimicrobial prescribing decision. ${ }^{14}$

Future studies should identify barriers and facilitators to recruiting personnel outside the traditional stewardship team

Affiliations: 1. Center of Innovation for Complex Chronic Healthcare, Edward Hines Jr VA Hospital, Hines, IL; 2. Department of Pharmacy Systems, Outcomes, and Policy, University of Illinois at Chicago, Chicago, IL; 3. Iowa City VA Health Care System, Iowa City, Iowa; 4. Division of Infectious Diseases, Department of Internal Medicine, University of Iowa Carver College of Medicine, Iowa City, Iowa; 5. VA Portland Healthcare System, Portland, Oregon; 6. VA Salt Lake City Health Care System and the University of Utah School of Medicine, Salt Lake City, Utah; 7. VA Pharmacy Benefits Management Services, Hines, Illinois; 8. University of Iowa Hospital and Clinics, Iowa City, Iowa; 9. Department of Geographical and Sustainability Sciences, College of Liberal Arts and Sciences, University of Iowa, Iowa City, Iowa; 10. University of Wisconsin School of Medicine and Public Health, Madison, Wisconsin and the William S. Middleton Memorial Veterans Hospital, Madison Wisconsin; 11. VA Greater Los Angeles Healthcare System and David Geffen School of Medicine at UCLA, Los Angeles, California.

${ }^{a}$ Authors with equal contribution.

Received December 1, 2017; accepted December 8, 2017

(C) 2018 by The Society for Healthcare Epidemiology of America. All rights reserved. 0899-823X/2018/3902-0010. DOI: 10.1017/ice.2017.299 
in the promotion of judicious antimicrobial prescribing. Depending on the clinical setting, these stewardship extenders may include non-ID pharmacists, hospitalists, and other ancillary staff, such as nursing. ${ }^{15-17}$ In resource-limited settings, the use of telemedicine or information technology may provide opportunities for external facilitation. ${ }^{2,18}$ For example, local personnel at a resource-limited hospital could interact with an ID specialist based at a different location. Infectious diseases physicians have provided HIV training to primary care and rural locations through the VA Specialty Care Access Network-Extension for Community (SCAN-ECHO) program. ${ }^{19}$

Optimal stewardship activities. Prospective audit-andfeedback and preauthorization are both effective strategies for antimicrobial stewardship within inpatient settings. ${ }^{2}$ Recent evidence suggests that prospective audit-and-feedback may be more effective than preauthorization in decreasing overall usage. ${ }^{20}$

Audit-and-feedback is based on a set of core principles, ${ }^{21}$ and the effectiveness of this strategy is dependent on how the feedback is delivered. ${ }^{22}$ Future studies should identify which methods for providing feedback are most effective for antimicrobial stewardship.

Using audit-and-feedback, ASPs can address multiple aspects of antimicrobial prescribing, including empiric prescribing, antimicrobial dosing, de-escalation, duration of therapy, and intravenous-to-oral formulation conversion. It is unclear which of these targets has the greatest potential impact on key stewardship outcomes, such as preventing the emergence of antimicrobial resistance, reducing CDI, minimizing other adverse events, and improving clinical outcomes. ASPs have limited resources and effort should be directed toward activities with the greatest potential impact.

As much as possible, stewardship processes should be incorporated into the daily workflow of frontline prescribers. ${ }^{2}$ Clinical decision support systems (CDSSs) may be helpful in promoting thoughtful prescribing at the point of care, particularly by informing empiric prescribing decisions and prompting prescribers to de-escalate or discontinue therapy. ${ }^{23,24}$ Further research on CDSSs should address not only the technical aspects of implementation but also the optimal methods for presenting complex data and antimicrobial recommendations in an understandable way. ${ }^{25}$

Many ASPs have partnered with microbiology labs to leverage rapid diagnostics and biomarkers to improve antimicrobial prescribing. ${ }^{26}$ Not only must these tests provide accurate and actionable results, but data must be standardized and results communicated effectively to change prescriber behavior. ${ }^{26}$ Developing better novel diagnostic tests and incorporating these tests into ASP efforts has great potential, because diagnostic uncertainty continues to be a major obstacle to appropriate antimicrobial use, especially in complex, dynamic environments such as critical care.

Evidence for antimicrobial dosing and duration. Standard antimicrobial doses are inadequate to meet pharmacokinetic and pharmacodynamic $(\mathrm{PK} / \mathrm{PD})$ targets in many patients. ${ }^{27,28}$ Data on what constitutes optimal dosing are limited, especially for certain populations (eg, obesity, critically ill) and categories of antimicrobials. Further research is needed.

Randomized-controlled trials have defined duration of antimicrobials for some infections, such as pneumonia and pyelonephritis, but for many infections, the recommended duration is based on expert opinion (eg, cystitis in men). ${ }^{29}$ If clinical trials and comparative effectiveness studies can provide robust data on the optimal duration of therapy for normative patients, it would facilitate stewardship efforts to ensure that antimicrobials are administered for no longer than necessary. Studies on duration of therapy are also needed in immunocompromised and other patients with complex comorbidities, who were often excluded from the aforementioned trials.

Stewardship metrics. We are at an early stage in understanding how best to evaluate stewardship processes and how these processes influence antimicrobial prescribing and clinical outcomes. In acute-care hospitals, antimicrobial days per 1,000 days present is currently the most commonly accepted metric for antimicrobial use. ${ }^{2}$ However, reasonable targets for reduction have yet to be established. These targets would need to account for differences in patient populations as well as differences in types of antimicrobials prescribed (eg, broad- vs narrow-spectrum agents).

The VHA has been working with the CDC to implement SAAR ratios as part of the antimicrobial use module within the National Healthcare Safety Network (NHSN). ${ }^{30}$ The SAAR is risk-adjusted for specific hospital characteristics and thereby represents an improvement over antimicrobial use metrics. However, the SAAR alone will not be sufficient in guiding more comprehensive evaluations at the facility level and in identifying institution-specific opportunities for improvement. ${ }^{31}$ SAARs do not provide data as to why antimicrobials are being prescribed (ie, use according to patient diagnosis) or how antimicrobials are being used (eg, number of patients exposed, rates and timing of de-escalation or overall duration of therapy). Additional metrics should provide actionable data to clinicians and stewardship teams on their antimicrobial prescribing behavior.

When making interfacility comparisons using metrics, inherent differences between hospitals must be considered. The SAAR attempts to adjust for some facility-level factors, such as facility bed size, number of intensive care unit beds, and facility medical school affiliation. ${ }^{30}$ Adjustments may also have to be made for differences in case mix, infection prevention practices, and antimicrobial resistance rates. The best strategies to account for these key determinants of antimicrobial use have yet to be defined.

An overarching goal of stewardship is the reduction of antimicrobial resistance; therefore, a major priority includes determining which metrics of antimicrobial use best predict patient- or population-level changes in antimicrobial resistance. Of course, the impact stewardship has on antimicrobial resistance will always be confounded by how successfully 
infection prevention practices prevent patient-to-patient transmission of resistant organisms.

Stewardship programs should also quantify clinical outcomes, including infection-related deaths and infectionrelated readmissions.

\section{Outpatient Antimicrobial Stewardship}

Optimal antimicrobial stewardship personnel. Guidelines for acute-care ASPs recommend that leaders of stewardship strategies should be ID specialists. ${ }^{2,13}$ However, few ID specialists are available in the outpatient sector. Therefore, it is of utmost importance to engage stewardship extenders in outpatient care settings (eg, community pharmacists, public health departments). Patients are also an important component of outpatient stewardship; patient demand is a known predictor for inappropriate antimicrobial prescribing. Data on methods by which to engage these stakeholders are limited. ${ }^{32,33}$ Research is also needed to identify strategies for external facilitation at the health system, payer, and/or federal level in the community.

Optimal stewardship activities. In the United States, most of the antimicrobial consumption occurs in the community, ${ }^{34}$ and at least $30 \%$ of prescribing is inappropriate. ${ }^{35}$ Stewardship strategies have been evaluated in the community, but most studies have focused on clinic-delivered interventions among primary care physicians. A systematic review demonstrated that communication training and point-of-care testing are effective in reducing unnecessary antimicrobial use in clinics, while education alone has failed to demonstrate a sustained benefit. ${ }^{5}$ Certain behavioral interventions have also reduced inappropriate antimicrobial use for acute respiratory tract infections. ${ }^{36}$ Continued evaluation of the impact of other stewardship strategies, such as the role of clinical decision support systems, delayed prescribing, and audit and feedback measures should be conducted. A major priority is the identification of barriers and facilitators to implementing effective strategies across all outpatient settings and sustaining their effectiveness over time. Additionally, the generalizability of stewardship strategies across all outpatient care settings (eg, emergency departments, urgent care, and independent practices and across large healthcare networks) are unknown.

Most stewardship evaluations have focused on the outcome of unnecessary antimicrobial-prescribing among clinic-based primary care physicians. However, few evaluate appropriate antimicrobial selection (eg, first-line agents) when antimicrobials are indicated (eg, symptomatic bacteriuria). Evidence is also needed to support the impact of specific stewardship interventions in the community, especially for specialty physicians, nonphysician providers, resource-limited settings and outpatient care delivered outside of the clinic setting (eg, emergency departments, urgent care).

Outpatient parenteral antimicrobial therapy (OPAT) is time intensive and is frequently outside the purview of many outpatient stewardship initiatives. As a first step, we recommend studies of existing OPAT practices in VHA.
Stewardship metrics. Little evidence is available to identify optimal stewardship metrics for outpatient settings. While audits to assess overall use have been recommended, ${ }^{37}$ it is unclear whether cases should be identified by International Classification of Disease, Tenth Revision (ICD-10) codes (syndrome-based approach) or antimicrobial prescriptions (agent-based approach). The impact of outpatient stewardship on antimicrobial resistance, CDI, and patient-centered outcomes has also not been definitively demonstrated, but research has suggested decreasing antimicrobial prescribing in outpatients has been associated with decreased resistance and CDI. ${ }^{38,39}$

The use of metrics for peer-to-peer and interfacility comparisons will have to account for differences between patient panels, which could potentially include differences in comorbidities and community resistance rates. Like metrics for inpatient interfacility comparisons, the best strategies to account for these key determinants of antimicrobial use have yet to be defined.

\section{Implementation of Antimicrobial Stewardship Across Inpatient and Outpatient Settings}

Recent regulatory mandates both in the VHA and outside the VHA should facilitate the expansion of inpatient stewardship efforts. While VHA stewardship activities in outpatient care settings are increasing, these interventions remain limited. ${ }^{40}$ Data are not available on the prevalence of stewardship interventions in outpatient care settings in the private sector, but it is likely that these efforts also remain limited. Barriers and facilitators to broader implementation of outpatient stewardship have not been identified. In addition, optimal timing of outpatient stewardship interventions and the role of inpatient ASP teams to facilitate stewardship in outpatient healthcare settings remains unknown.

Achieving the research targets discussed above for both inpatients and outpatients should facilitate efforts of widespread stewardship implementation. For example, clarifying which stewardship activities are the most impactful should help to implement processes in settings with limited resources and across large healthcare networks. Factors that ensure sustainability and scalability over time need to be identified.

\section{CONCLUSION}

In conclusion, a multidisciplinary group of VHA experts identified several research targets for antimicrobial stewardship. Pursuit of research in these areas should improve health care for veterans and the nation while continuing to address the crisis of antimicrobial resistance. Addressing these knowledge gaps in the VHA has the opportunity to be far-reaching because healthcare systems in the private sector can apply what was learned in VHA to their own stewardship programs and initiatives. With a robust data source and a partnership between clinical care and research, the VHA provides a unique opportunity to evaluate the implementation and impact of a national antimicrobial stewardship policy. 
TABLE 1. Assessment of Current Evidence and Recommendations for Research Needs in Antimicrobial Stewardship

\begin{tabular}{|c|c|c|}
\hline Topic & Current Evidence & Research Needs (Specific QUERI 6-step model addressed) \\
\hline $\begin{array}{l}\text { Inpatient antimicrob } \\
\text { Optimal structure of } \\
\text { ASP teams }\end{array}$ & $\begin{array}{l}\text { oial stewardship } \\
\text { ID specialists are effective leaders of ASPs, but there are not } \\
\text { enough ID specialists to provide on-site leadership across } \\
\text { all healthcare facilities. }\end{array}$ & $\begin{array}{l}\text { Identify barriers and facilitators to recruiting personnel } \\
\text { outside the traditional stewardship team (stewardship } \\
\text { extenders) to promote judicious antimicrobial- } \\
\text { prescribing (step 3) } \\
\text { Develop strategies for external facilitation in resource- } \\
\text { limited settings (steps } 4 \text { and 5) }\end{array}$ \\
\hline $\begin{array}{l}\text { Optimal } \\
\text { stewardship } \\
\text { activities }\end{array}$ & $\begin{array}{l}\text { Prospective audit-and-feedback and preauthorization are } \\
\text { core stewardship strategies. }\end{array}$ & $\begin{array}{l}\text { Identify the highest impact targets for audit-and-feedback } \\
\text { (steps } 2 \text { and } 3 \text { ) } \\
\text { Determine the optimal approach to providing feedback } \\
\text { (steps } 3 \text { and 5) } \\
\text { Develop new diagnostics to facilitate more rapid feedback } \\
\text { (step 1) }\end{array}$ \\
\hline Stewardship Metrics & $\begin{array}{l}\text { Days of therapy is the most commonly accepted metric for } \\
\text { inpatient antimicrobial use. } \\
\text { The SAAR is an early attempt to adjust for some facility } \\
\text { characteristics, thereby permitting benchmarking. }\end{array}$ & $\begin{array}{l}\text { Develop and validate metrics to guide more comprehensive } \\
\text { evaluations of antimicrobial-prescribing at the facility- } \\
\text { level and to provide actionable data for effective, } \\
\text { population-level stewardship interventions (step 2) } \\
\text { Methods for inter-facility risk-adjustment to enable } \\
\text { benchmarking are needed (step 2) }\end{array}$ \\
\hline
\end{tabular}

\section{Outpatient antimicrobial stewardship}

\begin{tabular}{|c|c|c|}
\hline $\begin{array}{l}\text { Optimal } \\
\text { antimicrobial } \\
\text { stewardship } \\
\text { personnel }\end{array}$ & Insufficent data to draw conclusions & $\begin{array}{l}\text { Identify and implement strategies to engage stewardship } \\
\text { extenders in the outpatient setting (steps } 4 \text { and 5) } \\
\text { Identify and implement strategies to conduct external } \\
\text { facilitation from the level of the health system (step 5) }\end{array}$ \\
\hline $\begin{array}{l}\text { Optimal } \\
\text { stewardship } \\
\text { activities }\end{array}$ & $\begin{array}{l}\text { Communication training, certain behavioral interventions, } \\
\text { academic detailing and point-of-care testing can help } \\
\text { reduce unnecessary antimicrobial use in clinics. }\end{array}$ & $\begin{array}{l}\text { Identify barriers and facilitators to implementing proven } \\
\text { strategies and sustaining their effectiveness over time (step 3) } \\
\text { Optimal combinations of stewardship strategies have not be } \\
\text { determined, especially in non-academic settings in the } \\
\text { absence of a research environment. (step 4) } \\
\text { Determine whether strategies that work in primary care are } \\
\text { also efficacious in other outpatient settings (steps } 2 \text { and 3) }\end{array}$ \\
\hline Stewardship metrics & Insufficent data to draw conclusions & $\begin{array}{l}\text { Develop and validate outpatient metrics (step 2) } \\
\text { Develop strategies to compare antimicrobial-prescribing } \\
\text { across providers/clinics while accounting for key } \\
\text { determinants of antimicrobial use (step 2) }\end{array}$ \\
\hline
\end{tabular}

NOTE. ID, infectious diseases; ASP, antimicrobial stewardship program; SAAR, standardized antimicrobial administration ratio.

\section{ACKNOWLEDGMENTS}

The views expressed in this article are those of the authors and do not necessarily reflect the position or policy of the Department of Veterans Affairs or the US government.

Financial support: This work was supported in part by funding from the VA Health Services Research and Development (HSR\&D) Service Center of Innovation (COIN) conference supplement for "Setting the Clinical Research
Agenda for MDROs in VA" (grant no. CIN 13-412) and VA QUERI CARRIAGE program (grant no. IP1 HX001993-01A1).

Potential conflicts of interest: All authors report no conflicts of interest or financial disclosures relevant to this article.

Address correspondence to Daniel Livorsi, MD, MSc, Assistant Professor, Division of Infectious Diseases, University of Iowa Carver College of Medicine, 
Iowa VA Health Care System, 601 Highway 6 West, Iowa City, IA 52246 (daniel-livorsi@uiowa.edu).

\section{REFERENCES}

1. Livermore DM. Minimising antibiotic resistance. Lancet Infect Dis 2005;5:450-459.

2. Barlam TF, Cosgrove SE, Abbo LM, et al. Implementing an antibiotic stewardship program: guidelines by the Infectious Diseases Society of America and the Society for Healthcare Epidemiology of America. Clin Infect Dis 2016;62:e51-e77.

3. Schuts EC, Hulscher ME, Mouton JW, et al. Current evidence on hospital antimicrobial stewardship objectives: a systematic review and meta-analysis. Lancet Infect Dis 2016;16:847-856.

4. Wagner B, Filice GA, Drekonja D, et al. Antimicrobial stewardship programs in inpatient hospital settings: a systematic review. Infect Control Hosp Epidemiol 2014;35:1209-1228.

5. Drekonja DM, Filice GA, Greer N, et al. Antimicrobial stewardship in outpatient settings: a systematic review. Infect Control Hosp Epidemiol 2015;36:142-152.

6. Chou AF, Graber CJ, Jones M, et al. Characteristics of antimicrobial stewardship programs at Veterans Affairs hospitals: results of a nationwide survey. Infect Control Hosp Epidemiol 2016;37:647-654.

7. Kelly AA, Jones MM, Echevarria KL, et al. A report of the efforts of the Veterans Health Administration National Antimicrobial Stewardship Initiative. Infect Control Hosp Epidemiol 2017; 38:513-520.

8. New antimicrobial stewardship standard. The Joint Commission website. https://www.jointcommission.org/assets/1/6/New_ Antimicrobial_Stewardship_Standard.pdf. Published 2016. Accessed April 28, 2017.

9. CMS issues proposed rule that prohibits discrimination, reduces hospital-acquired conditions, and promotes antibiotic stewardship in hospitals, 2016. Centers for Medicare and Medicaid Services website. https://www.cms.gov/Newsroom/MediaReleaseDatabase/ Fact-sheets/2016-Fact-sheets-items/2016-06-13.html. Published 2016. Accessed April 28, 2017.

10. Jones M, Huttner B, Madaras-Kelly $\mathrm{K}$, et al. Parenteral to oral conversion of fluoroquinolones: low-hanging fruit for antimicrobial stewardship programs? Infect Control Hosp Epidemiol 2012;33:362-367.

11. Huttner B, Jones M, Rubin MA, et al. Double trouble: how big a problem is redundant anaerobic antibiotic coverage in Veterans Affairs medical centres? J Antimicrob Chemother 2012;67:1537-1539.

12. Jones BE, Sauer B, Jones MM, et al. Variation in outpatient antibiotic prescribing for acute respiratory infections in the veteran population: a cross-sectional study. Ann Intern Med 2015;163:73-80.

13. US Department of Health and Human Services. Core elements of hospital antibiotic stewardship programs. Centers for Disease Control and Prevention website. https://www.cdc.gov/getsmart/ healthcare/pdfs/core-elements.pdf. Published 2014. Accessed April 28, 2017.

14. Echevarria K GJ, Kelly AA, Morreale AP, Neuhauser MM, Roselle G. Development and applicaiton of an objective staffing calculator for antimicrobial stewardship programs in the Veterans Health Administration. Am J Health-Syst Pharm 2017;74:e493-e498.
15. Yam P, Fales D, Jemison J, Gillum M, Bernstein $M$. Implementation of an antimicrobial stewardship program in a rural hospital. Am J Health-Syst Pharm 2012;69:1142-1148.

16. Trivedi KK, Kuper K. Hospital antimicrobial stewardship in the nonuniversity setting. Infect Dis Clin North Am 2014;28:281-289.

17. Olans RN, Olans RD, DeMaria A Jr. The critical role of the staff nurse in antimicrobial stewardship-unrecognized, but already there. Clin Infect Dis 2016;62:84-89.

18. Stenehjem E HA, Buckel WR, Jones PS, et al. Stewardship in Community Hospitals Optimizing Outcomes and Resources (SCORE): a cluster-randomized controlled trial investigating the impact of antibiotic stewardship in 15 small, community hospitals. Paper presented at IDWeek 2016, October 26-30, New Orleans, LA.

19. Moeckli J, Stewart KR, Ono S, et al. Mixed-methods study of uptake of the Extension for Community Health Outcomes (ECHO) telemedicine model for rural veterans with HIV. J Rural Health 2016;33:323-331.

20. Tamma PD, Avdic E, Keenan JF, et al. What is the more effective antibiotic stewardship intervention: pre-prescription authorization or post-prescription review with feedback? Clin Infect Dis 2017;64:537-543.

21. Brehaut JC, Colquhoun HL, Eva KW, et al. Practice feedback interventions: 15 suggestions for optimizing effectiveness. Ann Intern Med 2016;164:435-441.

22. Ivers N, Jamtvedt G, Flottorp S, et al. Audit and feedback: effects on professional practice and healthcare outcomes. Cochrane Database Syst Rev 2012:CD000259.

23. Forrest GN, Van Schooneveld TC, Kullar R, Schulz LT, Duong P, Postelnick M. Use of electronic health records and clinical decision support systems for antimicrobial stewardship. Clin Infect Dis 2014;59(Suppl 3):S122-S133.

24. Holstiege J, Mathes T, Pieper D. Effects of computer-aided clinical decision support systems in improving antibiotic prescribing by primary care providers: a systematic review. J Am Med Inform Assoc 2015;22:236-242.

25. Filice GA, Drekonja DM, Thurn JR, et al. Use of a computer decision support system and antimicrobial therapy appropriateness. Infect Control Hosp Epidemiol 2013;34:558-565.

26. Timbrook TT, Morton JB, McConeghy KW, Caffrey AR, Mylonakis E, LaPlante KL. The effect of molecular rapid diagnostic testing on clinical outcomes in bloodstream infections: a systematic review and meta-analysis. Clin Infect Dis 2017;64:15-23.

27. Theuretzbacher U. Pharmacokinetic and pharmacodynamic issues for antimicrobial therapy in patients with cancer. Clin Infect Dis 2012;54:1785-1792.

28. Roberts JA, Paul SK, Akova M, et al. DALI: defining antibiotic levels in intensive care unit patients: are current beta-lactam antibiotic doses sufficient for critically ill patients? Clin Infect Dis 2014;58:1072-1083.

29. Hayashi Y, Paterson DL. Strategies for reduction in duration of antibiotic use in hospitalized patients. Clin Infect Dis 2011;52:1232-1240.

30. Antimicrobial use and resistance module. Centers for Disease Control and Prevention website. https://www.cdc.gov/nhsn/pdfs/ pscmanual/11pscaurcurrent.pdf. Published 2017, Accessed November 27, 2017.

31. Moehring RW, Anderson DJ, Cochran RL, et al. Expert consensus on metrics to assess the impact of patient-level antimicrobial 
stewardship interventions in acute-care settings. Clin Infect Dis 2017;64:377-383.

32. Gonzales R, Steiner JF, Lum A, Barrett PH Jr. Decreasing antibiotic use in ambulatory practice: impact of a multidimensional intervention on the treatment of uncomplicated acute bronchitis in adults. JAMA 1999;281:1512-1519.

33. Little P, Stuart B, Francis N, et al. Effects of internet-based training on antibiotic prescribing rates for acute respiratory-tract infections: a multinational, cluster, randomised, factorial, controlled trial. Lancet 2013;382:1175-1182.

34. Suda KJ, Hicks LA, Roberts RM, Hunkler RJ, Danziger LH. A national evaluation of antibiotic expenditures by healthcare setting in the United States, 2009. J Antimicrob Chemother 2013;68:715-718.

35. Fleming-Dutra KE, Hersh AL, Shapiro DJ, et al. Prevalence of inappropriate antibiotic prescriptions among US ambulatory care visits, 2010-2011. JAMA 2016;315:1864-1873.
36. Meeker D, Linder JA, Fox CR, et al. Effect of behavioral interventions on inappropriate antibiotic prescribing among primary care practices: a randomized clinical trial. JAMA 2016;315:562-570.

37. Sanchez GV, Fleming-Dutra KE, Roberts RM, Hicks LA. Core elements of outpatient antibiotic stewardship. MMWR Recomm Rep 2016;65:1-12.

38. Dantes $\mathrm{R}, \mathrm{Mu} \mathrm{Y}$, Hicks LA, et al. Association between outpatient antibiotic prescribing practices and community-associated Clostridium difficile infection. Open Forum Infect Dis 2015;2:ofv113.

39. Seppala H, Klaukka T, Vuopio-Varkila J, et al. The effect of changes in the consumption of macrolide antibiotics on erythromycin resistance in group A streptococci in Finland. Finnish Study Group for Antimicrobial Resistance. N Eng J Med 1997;337:441-446.

40. Kelly A. The National Antimicrobial Stewardship Survey Report. Antimicrobial Stewardship Task Force Monthly Educational Webinar; July 22, 2016. 